\title{
The application of statistical methods to control the quality of the contact pairs of electrical connectors
}

\author{
Konstantin V. Podmasteryev ${ }^{1}$, Vladimir V. Markov ${ }^{1}$, and Anna V. Morozova, ${ }^{2, *}$ \\ ${ }^{1}$ Oryol State University name's of I.S. Tyrgenev, Department «Device-building, Mertology and Sertification», 302026 Orel 95 \\ Komsomolskaya Street, Russia \\ ${ }^{2}$ Bryansk State Technical University, Department Innovative Development of University, 241035 Bryansk 7, 50 let Oktyabrya bul., \\ Russia
}

\begin{abstract}
The development of electronic technology in the direction of improving its performance and speed, accompanied by the decrease of its dimensions and mass. This trend requires a new generation of electrical connectors differing from the basic models of a large number of contacts and density of placement on the insulator, having low contact resistance and high insulation resistance, high reliability and long life. The article describes the way of practical application of statistical methods for quality control of contact pairs of electrical connectors. The objects of study of effectiveness of statistical methods of quality management selected two typical details contact pairs received by turning on automatic lathes: pin and socket. The article considers the process of collecting statistical information about the technological process automatic turning of parts of the contact pairs. Shows the results of processing statistical information about the quality of the details of the contact pairs. The use of statistical methods for quality control of contact pairs of electrical connectors allows to correctly configure technological equipment according to the results of making a test batch of parts. Recommendations are made to improve the quality of the contact pairs of connectors. Compiled by technique of quality management of contact pairs according to the results of the statistical analysis of technological processes of their manufacture.
\end{abstract}

\section{Introduction}

The quality standard of products is determined by the perfection of technological processes of its manufacture $[5,6,8,9,23]$. The technological process of production is accompanied by a manifestation of many factors [11, $14,18,19,22]$ that vary randomly $[10,12,16,20,21]$. Therefore, to evaluate the degree of perfection of technological processes it is necessary to use statistical methods of information analysis [7, 13, 15, 17, 20]. Statistical methods allow us to estimate the basic properties of the process: willingness, precision, and stability. Typical examples of batch production are contact pairs of electrical connectors. They are manufactured in batch sizes from hundreds to tens of thousands of pieces. For the manufacture of parts of contact pairs are used by the automatic equipment. For example, parts of the "body of rotation" are manufactured by turning on lathes. Therefore, technological processes for the manufacture of contact pairs must obey the known laws of distribution of probabilities. The purpose of this paper is a summary of the methodology of application of statistical methods for quality control of contact pairs of electrical connectors and a demonstration of the results of experimental studies of the effectiveness of its application.

\section{Description of research objects}

The objects of study of effectiveness of statistical methods of quality management selected two typical details contact pairs received by turning on automatic lathes: pin and socket. Blanks for pin and socket contact pairs is brass rod, profile "round".

The manufacturing process of the contact pairs of rectangular electrical connectors consists of a complex of technological operations:

1) the complex of technological operations for blanking production, the purpose of which is to obtain workpieces of the type "Strip" of metal sheet materials or workpieces of the type "Rod" from the hire of round or hexagonal (depending on the design features of the pins and sockets contact pairs);

2) the complex of technological operations of primary production, the purpose of which is the shaping of parts of type "Pin" and "Socket" (two main details of the contact pairs); for the production of parts of type "Socket" is the basic method of forming is cold stamping, production of parts of type "Pin" can be used as cold stamping and lathe machine operation. Note that the processes of formation of pins and sockets are the main contributors to the formation of the electrical parameters and mechanical characteristics of the contact pairs collected in the connector;

\footnotetext{
* Corresponding author: niotiostu@gmail.com
} 
3) the complex of technological operations of applying a conductive coating on the surfaces of the contact pair by the method of electroplating. As coating materials are used metals with a low specific electrical resistance (silver, copper, gold, Nickel) and alloys with special electrical properties (alloy, "tin-bismuth" and others);

4) the complex of technological operations for the preparation of contact pairs for Assembly in the connector. The essence of this stage consists in removal of stamped pins and sockets with a common bandwidth. The peculiarity of the implementation of technological processes of preparation of contact pairs for Assembly lies in the subjective nature of the results due to the large number of manual operations performed by a forager connectors;

5) the complex of technological operations of the connector Assembly: Assembly of contact pairs in the insulator and secure them. These operations are performed using special jigs, so the results of the operation may be subject to the objective laws of distribution of probabilities.

To provide confidence in the required level of product quality, all manufacturing operations documented in the individual work instructions; data on progress are regularly recorded.

In the individual job description provides criteria for satisfactory completion of specific work and its compliance with quality standards. Standards of quality of performance of the work issued in the form of rules, posters, pictures or samples. Workplaces include products showing the worker the desired quality of performance of its work.

A mandatory element of the quality management system at this stage are inspection and testing. To reduce production losses from biased decisions seek to control quality of products at the points of greatest sensitivity to changes.

The manufacturing process of a pin contact pair involves turning processing of outer cylindrical surfaces, and cutting off the finished part from the bar. The object of measurement selected outer diameter of the finished pin, equal to $3.7 \mathrm{~mm}$ with a unilateral tolerance of body parts equal to $0.16 \mathrm{~mm}$.

The process of manufacture of socket contact pairs involves turning processing of inner and outer cylindrical surfaces, milling of flats and cutting of the finished part. The object of measurement selected inside diameter of the finished nest, equal to $8.14 \mathrm{~mm}$ with a unilateral tolerance of body parts, is equal to $0.06 \mathrm{~mm}$.

Turning parts contact pairs were produced on automatic lathe type "NEXTURN". This lathe machine has a numerical control system and resolution of the positioning of the parts to $0.001 \mathrm{~mm}$.

As means for measuring dimensions of parts of the contact pairs of connectors were used:

1) to measure the outside diameter of the pin is smooth micrometer with measuring range $0 . . .25 \mathrm{~mm}$ division value $0.001 \mathrm{~mm}$;

2) to measure the internal diameter of the socket shadow projector Nikon with measuring range $0 \ldots 300$ $\mathrm{mm}$ division value $0.001 \mathrm{~mm}$.
The control was subjected to two batches of the finished parts of the contact pairs. For each type of the details of the parties selected a sample size of 50 pieces. Selection of products for the size measurement was carried out continuously in the process of their manufacture on automatic lathe.

\section{The methodic of experiment}

To improve the objectivity of the process of collecting and processing statistical information on the quality of the parts and contact pairs atonement process equipment developed a methodology which includes several steps.

1 Analysis of working drawings of parts. According to the analysis of working drawings of the pin and socket of a selected size, which will be the basis of the statistical analysis of the disposition of equipment.

2 Preparation of measuring instruments. Measuring tools (micrometer smooth and the shadow projector) directly before the measurements were calibrated to determine the measurement error. The measurement error was determined by repeated measurements of the block size limit measures of the lengths corresponding to the size of parts of the contact pairs. For micrometer error made $\pm 0.004 \mathrm{~mm}$. For the shadow of the projector, the error amounted to $\pm 0.005 \mathrm{~mm}$.

3 The preparation of the equipment. Before the experiment lathe machine is configured for turning the set of parts and made a test piece. After making a trial of parts of lathe machine began to manufacture serial parts.

4 The conduct of the experiment. Measure the dimensions of the pin and socket were carried out at the operator's lathe machine. The finished part is removed from the hopper lathe machine, cleansed of lubricant and subjected to measurement. The measurement results recorded in the Protocol. Thus on each object of the studies are shown the measurement results of 50 pieces.

5 Processing of measurement results. Statistical processing of results of measurements of the dimensions of the parts conducted in the following order.

Determined the value of the configuration center, lathe machine, as mid-tolerance part size:

$$
m e=d_{\min }+\frac{T d}{2},
$$

where $m e$ - the median of the tolerance field size, $\mathrm{mm} ; d_{\min }$ - smallest allowable dimension value, $\mathrm{mm} ; \mathrm{Td}$ - dimension tolerance, $\mathrm{mm}$.

Estimated arithmetic mean value of dimensions:

$$
\bar{d}=\frac{\sum_{i=1}^{n} d_{i}}{n},
$$

where $d_{i}$ - the value of the diameter of the $i$-th part, $\mathrm{mm} ; n$-number of parts, amount of detail $(i=1 \ldots n)$.

The estimated standard deviation of the sizes: 


$$
S_{d}=\sqrt{\frac{\sum_{i=1}^{n}\left(d_{i}-\bar{d}\right)}{n-1}}
$$

Field size tolerance for each part is broken into intervals of width $0.01 \mathrm{~mm}$. Is the width of the intervals selected on the basis of the error measurement means, taking into account the safety factor $k=2$.

To determine the frequency of hit of values of dimensions of parts in each interval (to percent):

$$
p=\frac{n_{j}}{N} \cdot 100 \%,
$$

where $n_{j}$ - the number of values that fall in the $j$-th interval; $N$ - the total number of dimension values $(N=$ $50)$.

The calculated ratio between half the dimension tolerance of parts and standard deviation. In this relation, taking into account the hypothesis about the normal distribution of the dimensions of parts, using the table of standardized normal distribution defined by the probability of getting the size of the parts in the boundaries of tolerance and probability of presence in a batch of defective parts.

6 Create of histograms. The experimental data are processed using the formulas (1) to (4), histograms of the sizes of parts of the contact pairs. Coordinate histograms are: $\mathrm{x}$-axis - dimensions of parts are within tolerance; and the $y-$ axis the frequency of contact values of the sizes of items in each interval (to percent).

The histograms indicated: arithmetic mean and standard deviation of the size of the part; the border and the median (middle) of the tolerance field.

7 Analysis of mood and precision equipment. According to the histogram, the coordinates of the average size and the middle of the tolerance field determined by the correct setting of technological equipment.

Against half of the tolerance field of the part size to the standard deviation of the size determined by the accuracy of the technological process, as the probability of the presence in the party of defective products.

8 Formation of proposals to improve the quality of the contact pairs of electrical connectors. The results of the analysis of statistical data proposed action to improve the quality of the products.

As an example, in figures 1 and 2 show histograms of the distribution of sizes of pin and socket contact pairs of the electrical connector.

\section{Discussion of results}

Consider the results of processing statistical information about the quality of the parts of contact pairs of electrical connectors and attunement process equipment.

Figure 1 shows a histogram of the distribution of the dimensions of the pin contact pairs. According to statistical data, plotted on a histogram, it is possible to draw the following conclusions.

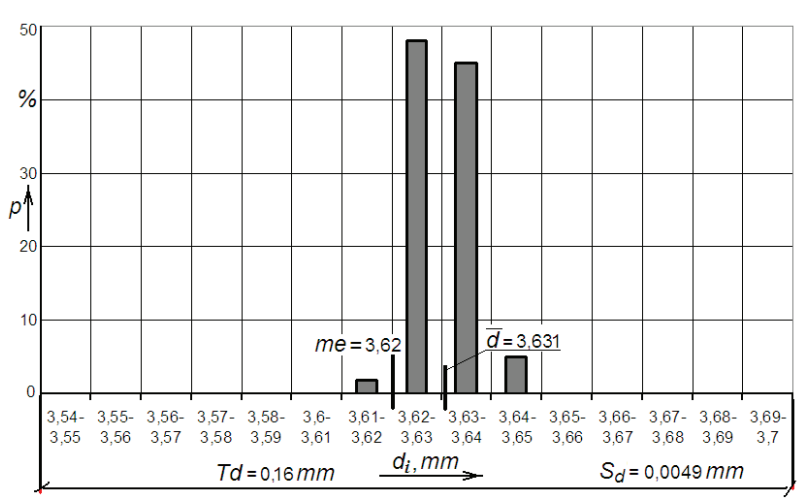

Fig. 1. A histogram of the distribution of the dimensions of the pin contact pair.

1 The shape of the histogram matches the normal distribution of probabilities. Statistical processing of results of measurements confirms this. Therefore, the manufacturing process automatic turning pin is stable.

2 The arithmetic average of the size of a pin does not coincide with the middle of the tolerance field size. It is offset $0.011 \mathrm{~mm}$ to the upper limit. Therefore, there is an increased probability of making the pins larger than the upper limit.

3 To improve the quality of the pins we recommend that you configure automatic lathe the size of $3.61 \mathrm{~mm}$ and not in the middle of the tolerance field ( $m e=3.62$ $\mathrm{mm}$ ). After this you need to make a trial batch of parts in an amount not less than 20 pieces to test the coincidence of the middle of the tolerance field and the mean value of the size of a pin.

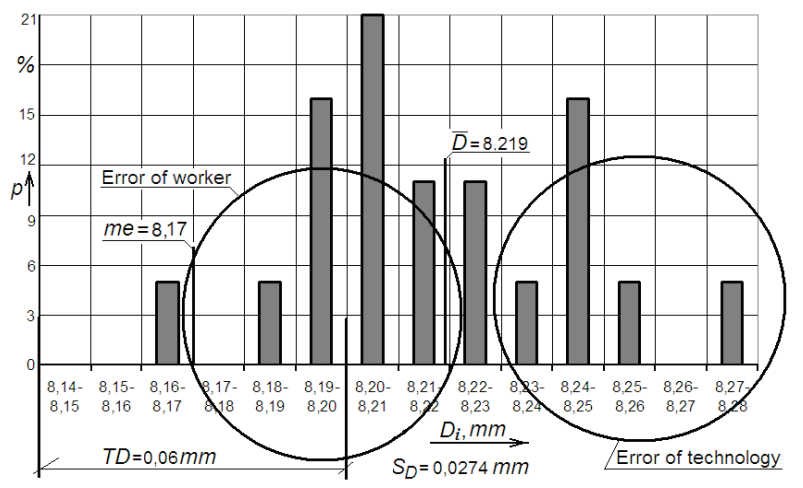

Fig. 2. Histogram of sizes of socket contact pairs.

4 Attitude is half of the size tolerance of the pin to the arithmetic mean is 16.36. In the table of the standardized normal distribution this corresponds to the probability of occurrence of defective products for size with a tolerance $T d=0.16 \mathrm{~mm}$, equal to $0.002 \%$. Utmost precision, this lathe machine is the size tolerance $T d_{M I N}=0.02 \mathrm{~mm}$; with this tolerance, the likely share of the marriage reaches $5 \%$. This means that this automatic lathe parts with tolerances less than $0.02 \mathrm{~mm}$ can be obtained only in the manufacture and subsequent sorting.

For this scheme, will analyze the technological process of manufacture of socket contact pairs. A histogram of the distribution of the dimensions of the slot shown in figure 2. According to statistical data, 
plotted on a histogram, it is possible to draw the following conclusions.

1 The shape of the histogram corresponds to a combination of two normal probability distribution, mathematical expectation which are offset from each other by about $0.04 \mathrm{~mm}$. This means that in the formation of the size of the pins involved two independent but stable random processes.

2 The arithmetic mean size of the nest did not significantly coincide with the middle of the tolerance field size. It is shifted by $0.05 \mathrm{~mm}$ to the upper limit, and this shift is almost equal to the dimension tolerance of the socket $(T d=0.06 \mathrm{~mm})$. From the histogram it is seen that this leads to appearance of defective parts that are larger than the upper size limit. The results of the calculation of the probability of occurrence of defective parts is set, it is equal to $19.71 \%$, and this kind of marriage is unrecoverable, as the study size of socket contact pairs internal.

3 To improve the quality of nests is necessary, first of all, to establish not one but two causes of defective parts. The first possible reason is incorrect configuration of lathe machine: it is set not to the middle of the size tolerance $(m e=8.17 \mathrm{~mm})$, while the upper size limit $\left(D_{\text {MAX }}=8.2 \mathrm{~mm}\right)$. The reason marriage provides the "burst" frequency of contact sizes in intervals in the range of 8.19 to $8.21 \mathrm{~mm}$. the Second reason is probably the deformation of a thin wall socket when it is boring lathe cutter. This reason gives the "burst" frequency of contact sizes in intervals in the range from 8.24 to 8.25 $\mathrm{mm}$.

Given the two possible causes of defective parts, to improve the quality of nests is recommended:

1) to configure automatic lathe to the middle of the tolerance field size - the size of $8.17 \mathrm{~mm}$;

2) to change the technological route, automatic turning of the socket so that the operation for boring the inside diameter were carried out to the outer grinding. This will ensure a thick wall when boring the inner diameter of the socket and, consequently, less deformation.

\section{Conclusions}

The use of statistical methods for quality control of contact pairs of electrical connectors allows to correctly configuring technological equipment according to the results of making a test batch of parts. Then the probability of occurrence of defective products at the stage of serial production of parts can be significantly reduced.

The authors consider it their pleasant duty to express my gratitude to the workers and engineering-technical workers of JSC "Karachevsky factory "Electrodetail" for useful participation, greater technical assistance in performing experiments, moral support and valuable advice.

\section{References}

1. V.E. Gmurman, Theory of probabilities and mathematical statistics (Higher School, Moscow, 1999)

2. V.V. Efimov, Statistical methods in the control quality of production (KnoRus, Moscow, 2013)

3. G.I. Utkin, Fundamental and Applied Problems of Equipment and Technology 6, 114 (2009)

4. S.F. Korndorf, Fundamental and Applied Problems of Equipment and Technology 1, 78 (2010)

5. S. R. Bowling, European Journal of Operational Research 159(3), 636 (2004)

6. C. H. Chen, M. B. C. Khoo, Computers \& Industrial Engineering 57(3), 1080 (2009)

7. P. Winkel, N. F. Zhang, Scandinavian Journal of Clinical and Laboratory Investigation 64(3), 195 (2004)

8. S. Bandini, F. Sartori, Artificial Intelligence for Engineering Design, Analysis and Manufacturing, 24(01), 17 (2010)

9. J. Sun, International Journal of production economics 114 (1), 149 (2008)

10. P. Schäffler, W. Rajner, Advanced Engineering Materials 6(6), 452 (2004)

11. Y. H. Chun, IIE Transactions 41 (9), 831 (2009)

12. C. H. Chen, Journal of Quality and Technical 17(2), 99 (2010)

13. Y. Zhang, Y. Teng, Y. Zhang, Chemical Engineering Science 65(6), 2153 (2010)

14. E. Hull, K. Jackson, J. Dick, Requirements engineering (Springer Science \& Business Media, 2010)

15. L. Huber, Validation of analytical methods (Agilent Technologies. Germany, 2010)

16. D. E. Seborg, Process dynamics and control (John Wiley \& Sons, 2010)

17. D. C. Montgomery, Statistical quality control, 7 (2009)

18. D. Reay, C. Ramshaw, A. Harvey, Process Intensification: Engineering for efficiency, sustainability and flexibility (ButterworthHeinemann, 2013)

19. J. R. Evans, W. M. Lindsay, Managing for quality and performance excellence (Cengage Learning, 2013)

20. L. D. Miles, Techniques of value analysis and engineering (Miles Value Foundation, 2015)

21. M. A. Razzhivina, B. A. Yakimovich, A. I. Korshunov, Pollack Periodica 10(2), 17 (2015)

22. S. N. Grigoriev, Measurement Techniques 11, 1311 (2013)

23. R. Shankar, M. Sundararajan, Indian Journal of Science and Technology, 8(15) (2015) 